\title{
Repensando la asignatura de Inglés en educación primaria a través del PTL
}

Recepción: 12/05/2020 | Revisión: 02/07/2020 | Aceptación: 27/08/2020 | Preprint: 10/12/2020 | Publicación: 01/10/2021

\author{
Miriam IDRISSI-CAO \\ Universidad de Valladolid \\ misicao.miriam@gmail.com \\ https://orcid.org/0000-0002-0972-3198
}

(iD) Natalia BARRANCO-IZQUIERDO

Universidad de Valladolid

natalia.barranco@uva.es

https://orcid.org/oooo-0002-7831-6158

\author{
Eduardo FERNÁNDEZ RODRÍGUEZ \\ Universidad de Valladolid \\ edufern@pdg.uva.es \\ https://orcid.org/0000-0001-8611-2510
}

\begin{abstract}
Resumen: En la última década, el inglés ha adquirido gran relevancia en el contexto educativo, relevancia que se puede comprobar por el elevado número de centros educativos que han pasado a ser secciones bilingües. El uso de metodologías de integración de contenido y lenguas está en auge y se moviliza en la impartición en lengua extranjera de disciplinas no lingüísticas. Sin embargo, en este proceso, la investigación y renovación del inglés como asignatura parece haber quedado paralizada en el tiempo. Hay que volver a mirar esta área incorporando los avances de metodologías más recientes, así como las propuestas de las políticas lingüísticas europeas. El objetivo de este trabajo es analizar los resultados de la puesta en práctica de una propuesta didáctica planificada con base en el enfoque Pluriliteracies approach to teaching for learning (PTL) desarrollado por Meyer, Halbach y Coyle (2015).
\end{abstract}

Palabras clave: enfoque PTL; lengua extranjera inglés; Educación Primaria.

\section{RETHINKING THE ENGLISH SUBJECT IN PRIMARY EDUCATION THROUGH PTL}

Abstract: In the last decade, English has acquired great relevance in the educational context. This relevance can be verified by the opening of a high number of bilingual schools. The application of content and language integration methodologies is rising and is carried out through the teaching of non-linguistic disciplines in a foreign language. Nevertheless, in this process, the research and renovation of English as a subject seem to have been at a standstill. This area must be reexamined adding the latest methodological advances and European language policies. The main goal of this paper is, therefore, to analyse the results of the implementation of a learning unit built on the basis of the PTL approach developed by Meyer, Halbach and Coyle (2015).

Keywords: PTL approach; English as a Foreign Language; Primary Education. 


\section{REPENSANT L'ASSIGNATURA D'ANGLÈS A L'EDUCACIÓ PRIMÀRIA A TRAVÉS DEL PTL}

Resum: Durant l'última dècada, l'anglès ha adquirit gran rellevància en el context educatiu, rellevància que es pot comprovar per l'elevat nombre de centres educatius que han passat a ser bilingües. L'ús de metodologies d'integració de contingut i llengües està en auge i es mobilitza en la impartició en llengua estrangera de disciplines no lingüístiques. No obstant això, la investigació i renovació de l'anglès sembla que s'hagi aturat en el temps. S'ha de tornar a veure aquesta àrea incorporant els avenços de metodologia més recents, així com les propostes de les polítiques lingüístiques europees. L'objectiu d'aquest treball és analitzar els resultats de la posada en pràctica d'una proposta didàctica planificada basada en l'enfocament Pluriliteracies approach to teaching for learning (PTL) desenvolupat per Meyer, Halbach i Coyle (2015).

Paraules clau: enfocament PTL; llengua estrangera anglès; Educació Primària.

\section{Introducción}

Las políticas lingüísticas educativas cuentan con una extensa trayectoria dentro de la historia de la Unión Europea. El Parlamento y el Consejo Europeo nombraron el 2001 como el Año Internacional de las Lenguas y sale publicado el documento titulado Common European Framework of Reference for Languages: Learning, Teaching, Assessment. La versión en español, Marco Común Europeo de Referencia para las lenguas: Aprendizaje, enseñanza, evaluación (en adelante, MCER), se publica en 2002, y supuso una gran diferencia en el modo de proceder de estas políticas lingüísticas europeas.

Para implantar las premisas del MCER (2002) sobre la enseñanza, aprendizaje y evaluación de lenguas, surgen nuevas metodologías y enfoques, entre ellas CLIL (Content and Language Integrated Learning), definido por Coyle, Hood y Marsh (2010) como un enfoque educativo dual que pone el foco en el uso de una lengua diferente a la materna para enseñar contenido y lengua. Se busca integrar ambas cuestiones para reforzar la idea de conectar a los estudiantes con el uso de diferentes idiomas en distintos momentos y con distintos propósitos (Coyle, 2015). A pesar de los múltiples beneficios que se han encontrado en torno a esta metodología (Bernaus et al., 2011), con el paso del tiempo y su puesta en práctica se ha comenzado a cuestionar la aplicación que se lleva a cabo en las aulas. Según Pérez Cañado (2016) CLIL contó con un periodo inicial de auge que, en la última media década, ha sido sustituido por un periodo de mayor debate y crítica.

Así, en 2015 sale a la luz el enfoque PTL o "Pluriliteracies approach to teaching for learning" desarrollado por Meyer, Halbach y Coyle dentro de un grupo de investigación conocido como The Graz Group. Este enfoque intenta crecer desde CLIL, abogando por la integración entre contenido y lengua, pero solventando los obstáculos encontrados en él. De este modo, la atención pasa a centrarse en dos ideas fundamentales: deep learning y literacidad.

A pesar de estos cambios, Halbach afirma que desde hace años «vivimos en tiempos de CLIL» $(2019$, p. 7) y, mientras asignaturas como ciencias sociales, ciencias de la naturaleza o tutoría se imparten en inglés, la asignatura de inglés y su investigación parecen relegadas a un segundo plano. Esta misma autora defiende la necesidad de alejarse de modelos gramaticales y de estudio exhaustivo de vocabulario para utilizar el inglés como herramienta de aprendizaje coincidiendo así con enfoques como CLIL o PTL. No obstante, afirma que las clases de inglés no pueden convertirse en una extensión de las clases de contenido. Esta asignatura sigue ocupando un lugar relevante en 
las aulas de la educación obligatoria, tiene una naturaleza diferente con su realidad propia y hay que cuidarla de manera específica (Halbach, 2019).

Es cierto que un mayor tiempo de exposición a la lengua -objetivo fundamental que persiguen las metodologías de integración de contenido y lengua- supone una mejora cuantitativa del tiempo empleado en su aprendizaje. No obstante, sería necesario reflexionar sobre su capacidad para impulsar una mejora cualitativa de dicho tiempo.

En este estudio queremos analizar la viabilidad de incluir los avances de la investigación en didáctica de las lenguas extranjeras en la propia asignatura de inglés. Se pretende valorar si las propuestas desarrolladas por modelos como PTL tienen cabida en las aulas de idioma y si utilizarlas como referencia mejora la actuación de los docentes. A través del análisis de una secuencia didáctica, tratamos de encontrar los puntos de unión entre las metodologías más actuales de enseñanza de segundas lenguas y otros principios y modelos aplicables a este contexto.

\section{Enfoque PTL}

El enfoque PTL coloca la literacidad de una asignatura en más de una lengua en la raíz del aprendizaje. De este modo, el objetivo fundamental es favorecer que el alumnado alcance un aprendizaje profundo (deep learning) que le permita desarrollar destrezas transferibles a diversas situaciones y que a su vez sirva para comunicar con éxito su conocimiento a través de diferentes culturas y lenguas (Meyer, Halbach y Coyle, 2015).

De esta definición extraemos dos cuestiones fundamentales que resulta interesante desarrollar en mayor detalle. Por un lado, hablamos de deep learning, definido por Meyer y Coyle (2017) como la asimilación de contenidos conceptuales, más la automatización de procedimientos, destrezas y estrategias asociadas a una asignatura. Se intenta ir más allá de la transmisión de contenidos propuesta por CLIL para hablar de la capacidad del idioma para crear significados (Coyle, 2015). La intención es que el alumnado sea capaz de utilizar o transferir lo aprendido a situaciones nuevas. Por otro lado, también adquiere un rol protagonista la literacidad, entendida como la construcción del discurso propio de una asignatura, en términos de códigos, géneros, funciones y roles, como garantía del progreso del conocimiento en sí mismo (Meyer, Coyle, Halbach y Schuck, 2015).

Para desarrollar esta literacidad, estos autores determinan que es necesario unir dos elementos fundamentales: continuo conceptual y continuo comunicativo, en su terminología en inglés conceptual continuum y communicating continuum. De esta manera, para avanzar en el conocimiento de una asignatura (conceptual continuum) es necesario profundizar en su discurso específico (communicating continuum). La segunda lengua, en este caso, se adapta a la situación, contexto o propósito con el que se usa, más que al conocimiento en sí mismo.

Atendiendo al esquema planteado, la idea de deep learning sería puente de unión entre ambas dimensiones. El deep learning se puede asociar con el modo en el que la lengua construye nuestra experiencia del mundo y nos permite compartirla con los otros. Así, lo que pretendemos a través del lenguaje es estimular las mentes del alumnado para acomodar los nuevos conocimientos 
sobre el mundo a aquellos otros que ya tienen. Dalton-Puffer (2013) habla de transformar los procesos de pensamiento en elementos análogos pero observables y, en ese aspecto, lo más práctico que encontramos son las funciones cognitivas del discurso (cognitive discourse functions, en adelante CDF). Las CDF pueden entenderse como los bloques que permiten la construcción del pensamiento, ayudando a estructurarlo, demostrarlo y comunicarlo y que, por tanto, constituyen la estructura básica del deep learning.

Con respecto a esta situación Dalton-Puffer (2013) desarrolla un constructo que relaciona una serie de intenciones comunicativas prototípicas con los pasos cognitivos necesarios (CDF) para gestionar el conocimiento. En esta línea, esta autora define que existen 7 tipos distintos de CDF a los que etiqueta de una manera difusa. Estas etiquetas son palabras de uso común en inglés, lo que supone que, como cualquier otra palabra, sus significados no son estancos, sino que se establecen a través de redes que se activan según los contextos. Los 7 tipos resultantes serían: 1. Classify; 2. Define; 3. Describe; 4. Evaluate; 5. Explain; 6. Explore; 7. Report. Se establece así que cada asignatura estimula la activación de estas CDF de una manera especializada a través de la implementación de lo que se conoce como dominio de actividad (Graz Group, 2016). Es decir, las CDF se estimulan a través de los modos concretos en los que cada asignatura procura "hacer, organizar, explicar y argumentar" su contenido.

Por último, ambas cuestiones quedan englobadas a través del uso de diferentes géneros o tipos de textos que, atendiendo a sus rasgos gramaticales, resultan característicos de contextos específicos (Schleppegrell, 2001). En nuestro caso, el contexto educativo y, de un modo más concreto, el inglés como asignatura.

En 2018, Meyer, Coyle, Imhof y Connolly revisan el trabajo anterior para añadir ciertos elementos obviados hasta el momento. Las aportaciones de Meyer, Rose y Gordon (2014) en el Universal Design for Learning (en adelante UDL) resultan interesantes para completar el enfoque PTL. Esta propuesta que se añade se basa en la búsqueda de un marco que ayude a optimizar la educación desde el análisis científico sobre el modo en el que aprenden los humanos. Estos autores hablan de que la educación debe buscar el desarrollo de personas "expertas en aprender" que: tengan un propósito y estén motivadas; capaces de buscar recursos y dominar el conocimiento; estratégicas y orientadas a objetivos. Para lograr esto, UDL plantea tres principios que todo aprendizaje debe tener bien definidos: por qué aprender (engagement); qué aprender (representation) y cómo aprender (action and expression). Todo ello, aportando también indicaciones sobre cómo mejorar el acceso (access), la construcción (build) y la internalización (internalize) del aprendizaje del alumnado (Meyer et al., 2014).

De este modo, Meyer et al. (2018) incorporan al proceso de construcción de conocimiento y significados a través del lenguaje, el compromiso con las emociones, la flexibilidad y la personalización puestas en valor por el modelo UDL.

\section{Planificando la asignatura de inglés a través del enfoque PTL}

El marco de esta experiencia se encuentra en la puesta en práctica de una secuencia didáctica que, teniendo en cuenta los elementos teóricos descritos con anterioridad, pretende dar luz a la 
posibilidad de programar asimilando las ideas propias de enfoques de integración en la asignatura de inglés en sí misma. Esta secuencia didáctica ha sido diseñada tomando como referencia la realidad de un centro educativo en particular. Aunque la viabilidad de replicar lo aquí descrito en otros contextos es factible, este centro conocido como Escuela Ideo cuenta con una serie de características específicas de interés que aportan sentido a la propuesta educativa que se analiza. Es por ello que, a continuación, se describe brevemente dicho contexto.

\subsection{Contexto}

Escuela Ideo ${ }^{1}$ es un centro educativo privado, laico, plurilingüe, mixto y de integración. Actualmente cuenta con línea tres desde los 2 años hasta los 18, aunque sigue expandiéndose año tras año.

El colegio se encuentra situado en el Km 0.500, Carretera de Colmenar a Alcobendas en el término municipal de Madrid, en un espacio remodelado y ajustado a los planteamientos esenciales de la escuela. En relación con las características de las familias, en general, se trata de familias jóvenes con un poder adquisitivo medio alto cuya orientación profesional se ubica en el sector servicios.

El centro tiene una serie de características que aportan personalidad propia al proyecto educativo. Entre las más destacables encontramos el trabajo por proyectos, importancia de las excursiones y el deporte, no hay uniforme, no se utilizan libros de texto ni se proponen exámenes, deberes o castigos. El centro confía en la capacidad de toda la comunidad educativa para trabajar en equipo y ofrecer al alumnado multitud de oportunidades de aprendizaje dentro y fuera del aula.

A nivel curricular, uno de los elementos más destacables es la combinación de las asignaturas de Ciencias Naturales y Ciencias Sociales en un único espacio curricular denominado Proyectos. Con una dedicación de 3 horas semanales, estas dos asignaturas se trabajan de una manera holística bajo un hilo argumental que va uniendo los contenidos. Dentro de su horario, el resto de asignaturas dedica un espacio a complementar el trabajo de Proyectos desde las características específicas de su disciplina, sin quitar o solapar el propio de las ciencias. Es decir, el resto de asignaturas ambienta parte de sus contenidos propios en la temática trabajada desde Proyectos, pero no asume ningún contenido que pertenezca al ámbito de las ciencias.

La secuencia didáctica que se analiza en el presente artículo gira en torno a la práctica docente dentro de la asignatura de Inglés de $5^{\circ}$ de Primaria. Por política del centro, en Primaria, la asignatura de Inglés cuenta con una carga lectiva de 8 horas semanales. Sin embargo, en los cursos de $5^{\circ}$ y $6^{\circ}$ de Primaria, dicha carga se reduce contando con un total de 6 horas semanales. Este cambio se debe a la introducción de una nueva asignatura conocida como "Digital Competence" que se imparte en inglés y que dentro del horario ha asumido esas dos horas restantes que harían falta para completar las 8 horas. Dentro de las horas de trabajo, el departamento de idiomas establece que:

- Un 50\% del tiempo debe destinarse al trabajo de los recursos de Milton. Inglés es la única área que cuenta con libro de texto utilizado como banco de recursos. A pesar de ello, su uso es sugerido y nunca debe superar más de ese $50 \%$.

- Dos horas dedicadas a complementar el hilo argumental que se esté trabajando en Proyectos.

- Una hora para actividades de interés específico del trimestre (animación a la lectura, festividades, concursos de deletreo...).

1 Escuela Ideo: https://www.escuelaideo.edu.es/ 
Teniendo en cuenta que el presente artículo analiza la potencialidad del enfoque PTL para ser integrado en Inglés, la labor de investigación se desarrolla en la propuesta planteada para las dos horas destinadas a complementar Proyectos. Se ha seleccionado esta franja de trabajo porque aporta un contenido que, sin ser el propio de una asignatura bilingüe como el caso de "Science", continúa siendo significativo para analizar las características del PTL. La temporalización para la secuencia didáctica fue la siguiente: 5 semanas de aplicación con una dedicación de 2 horas por semana, dando lugar a un total de 10 horas. Los contenidos asociados a la secuencia didáctica se enmarcaron dentro del proyecto 3 del curso titulado "Ministerio del Tiempo", que daba apoyo a un proyecto de Ciencias Sociales sobre historia.

\subsection{Elementos clave en el desarrollo de la secuencia didáctica}

Atendiendo a la temporalización, durante esas semanas, se estableció que las horas destinadas a proyectos de la asignatura inglés estuvieran programadas siguiendo las características del enfoque PTL. A la hora de preparar la secuencia didáctica, se analizaron las características de esta metodología y el modo en el que podían encajarse en el aula. Así, el desarrollo didáctico buscó atender a las cuestiones planteadas en la Figura 1.

\begin{tabular}{|c|c|c|}
\hline $\begin{array}{l}\text { GENERATING \& SUSTAINING } \\
\text { COMMINTMENT AND } \\
\text { ACHIEVEMENT } \\
\text {-Engagement- (Why) } \\
\text { Se busca que las actividades propuestas } \\
\text { en la práctica estimulen el crecimiento } \\
\text { personal del alumnado en términos de } \\
\text { afecto, compromiso, dominio y reflexión. } \\
\text { Todas ellas deben quedar } \\
\text { interrelacionadas entre sí y, a su vez, } \\
\text { englobar a los agentes principales del } \\
\text { proceso de aprendizaje, es decir, docente } \\
\text { y alumnado. Es fundamental que los } \\
\text { docentes sean capaces de definir los } \\
\text { objetivos de aprendizaje en términos de } \\
\text { cuatro aspectos relevantes: }\end{array}$ & $\begin{array}{l}\text { DEMONSTRATING \& } \\
\text { COMMUNICATING UNDERSTANDING } \\
\text {-Representation-(What) } \\
\text { Relevancia y presencia de la lengua como } \\
\text { herramienta básica para lograr un aprendizaje } \\
\text { profundo de la asignatura. } \\
\text { Para comunicar su aprendizaje el alumnado } \\
\text { debe identificar adecuadamente el propósito y } \\
\text { su audiencia, haciendo las elecciones correctas } \\
\text { en relación con el modo, el género y estilo del } \\
\text { mensaje. Así, en las actividades que se } \\
\text { presenten, se debe lograr que el alumnado sea } \\
\text { consciente de: }\end{array}$ & $\begin{array}{l}\text { MENTORING LEARNING AND } \\
\text { PERSONAL GROWTH } \\
\text {-Action \& Expression- (How) } \\
\text { El docente debe ser capaz de orquestar los } \\
\text { tres elementos anteriores presentes en el } \\
\text { modelo PTL. }\end{array}$ \\
\hline $\begin{array}{l}\text { Affect: Access } \\
\text { Hacer evidentes objetivos afectivos tales } \\
\text { como actitudes, eficacia personal, } \\
\text { motivación y bienestar. La predisposición } \\
\text { del alumnado a esforzarse en su } \\
\text { aprendizaje viene determinada por la } \\
\text { motivación que se genera y la medida en } \\
\text { la que se satisfacen sus necesidades. } \\
\text { Engagement: Access } \\
\text { La idea de "engagement" o compromiso } \\
\text { es un constructo formado por múltiples } \\
\text { dimensiones. Entre las más relevantes } \\
\text { Wang et al. (2016) hablan del } \\
\text { compromiso cognitivo (hacia el } \\
\text { procesamiento de la información), de } \\
\text { comportamiento (esfuerzo hacia las } \\
\text { tareas), el emocional (interés) y el social } \\
\text { (en el trabajo con el resto de } \\
\text { compañeros/as). Estas dimensiones cada } \\
\text { alumno/a las combinas de diferentes } \\
\text { maneras y, al mezclarse, provocan el } \\
\text { desarrollo de hábitos específicos de } \\
\text { aprendizaje. } \\
\text { Mastery: Build } \\
\text { La intención es que las clases favorezcan } \\
\text { el dominio del conocimiento y destrezas } \\
\text { con una dificultad progresiva. Construir } \\
\text { objetivos de aprendizaje consecutivos que } \\
\text { permitan al alumno/a ir analizando su } \\
\text { progreso y comprendiendo los procesos } \\
\text { involucrados para alcanzar el éxito en las } \\
\text { tareas. } \\
\text { Reflection: Internalise } \\
\text { Espacio que las clases aportan al } \\
\text { alumnado para establecer objetivos de } \\
\text { aprendizaje apropiados, asi como los } \\
\text { caminos para conseguirlos. } \\
\text { Monitorizar el proceso de aprendizaje, } \\
\text { guiarlo y adaptarlo en situaciones en las } \\
\text { que se presenten dificultades. }\end{array}$ & $\begin{array}{l}\text { Purpose: Intención comunicativa presente en la } \\
\text { actividad. } \\
\text { Genre: Modos en los que, atendiendo a las } \\
\text { convenciones culturales, se comunica el } \\
\text { conocimiento. } \\
\text { Mode: Input, es decir, aquellos recursos e } \\
\text { idioma al que se expone al alumnado para dar } \\
\text { lugar a su aprendizaje. } \\
\text { Style: Estilo elegido para desarrollar los } \\
\text { intercambios commicativos (formal/informal). } \\
\text { ACTIVATING PRIOR KNOWLEDGE, } \\
\text { CONCEPTUALISING \& REFINING } \\
\quad \text { SKILLS } \\
\text {-Representation- (What) } \\
\text { De qué manera se está trabajando para que las } \\
\text { sesiones partan de los conocimientos previos } \\
\text { del alumnado y si hay una internalización } \\
\text { progresiva de los conceptos que ayude a } \\
\text { desarrollar nuevas destrezas y mejorar las ya } \\
\text { existentes. } \\
\text { Para construir aprendizaje, el alumnado debe } \\
\text { ser capaz de utilizar estrategias y destrezas para } \\
\text { transformar los hechos en conocimiento } \\
\text { conceptual siguiendo los procesos específicos } \\
\text { de cada asignatura. } \\
\text { Atender a que exista una presencia clara de las } \\
\text { cuestiones necesarias para alcanzar un } \\
\text { conocimiento profundo sobre la asignatura: } \\
\text { Facts: Los elementos básicos que un alumno/a } \\
\text { debe conocer para familiarizarse con una } \\
\text { asignatura o resolver problemas en ella. } \\
\text { Concepts: Las interrelaciones existentes entre } \\
\text { los elementos básicos que se encuentran en una } \\
\text { estructura más amplia que hace que funcionen } \\
\text { juntos. } \\
\text { Procedures \& Strategies: Conocimiento sobre } \\
\text { cómo hacer las cosas, métodos de investigación } \\
\text { y criterios para utilizar las técnicas que sirvan } \\
\text { de puente para comprender los dos elementos } \\
\text { anteriores. }\end{array}$ & $\begin{array}{l}\text { Designing \& Evaluating: } \\
\text { Capacidad docente de manejar capacidades } \\
\text { pedagógicas sofisticadas, es decir, dominar } \\
\text { un variado repertorio de estrategias de } \\
\text { enseñanza y evaluación continua sobre el } \\
\text { momento de aprendizaje en el que se } \\
\text { encuentra el alumnado. } \\
\text { El docente debe facilitar y activar el } \\
\text { aprendizaje más que al que aprende. } \\
\text { Generar caminos de aprendizaje negociados } \\
\text { de manera conjunta con el alumnado para } \\
\text { que aprenda sobre sí mismo y } \\
\text { continuamente reflexione sobre su } \\
\text { progreso. } \\
\text { Scaffolding: } \\
\text { Encontrar el mejor modo de proporcionar } \\
\text { las mejores oportunidades de aprendizaje. } \\
\text { Diseño y rediseño de tareas y de secuencias } \\
\text { para que el aprendizaje sea visible para el } \\
\text { alumnado y el docente. } \\
\text { Uso de distintas estrategias para apoyar el } \\
\text { aprendizaje. Proporcionar múltiples modos } \\
\text { para comprometerse con la tarea. } \\
\text { Entornos flexibles y que respondan al } \\
\text { alumnado ayudándole a crecer en } \\
\text { compromiso y resiliencia. } \\
\text { Feedback: } \\
\text { Debe existir feedback constante para que el } \\
\text { alumnado se comprometa con la definición } \\
\text { y redefinición de los objetivos de } \\
\text { aprendizaje que se proponen. } \\
\text { Se debe proporcionar al alumnado la } \\
\text { capacidad de evaluar su propio trabajo } \\
\text { guiado por el profesor. } \\
\text { Assessment: } \\
\text { Que ayude al alumnado a seguir } \\
\text { formándose y sume en su proceso de } \\
\text { aprendizaje. }\end{array}$ \\
\hline
\end{tabular}

Figura 1. Pautas para el desarrollo de una secuencia didáctica en el aula de inglés. Fuente: Propia a partir de Meyer et al. (2014)y Meyer et al. (2018). 


\section{Método}

La experiencia que aquí se presenta se adscribe al marco metodológico de la investigación descriptiva, incluyendo dentro del mismo estudio valores cuantitativos y cualitativos. De este modo, la intención es describir las características que adquiere la asignatura de Inglés en relación con las actividades comunicativas de la lengua, funciones cognitivas del discurso, dominios de actividad y géneros al ser programada en torno a PTL.

\subsection{Codificación}

Para realizar este análisis contamos con la Figura 2, donde aparecen las sesiones y actividades de la secuencia didáctica y la Figura 3, que asocia estas actividades con los componentes de PTL. Finalmente, con la intención de analizar los elementos descritos en las Figuras 2 y 3 , se muestra una radiografía de la secuencia didáctica codificada en los siguientes términos:

\section{En la Figura 2:}

En la primera columna aparecen letras de la A a la J, siendo cada una de ellas una sesión de cincuenta minutos. Cada letra va acompañada del nombre que recibe dicha sesión.

En la segunda columna encontramos números desde 1 a un máximo de 4, siendo cada uno de ellos el número de cada actividad de la sesión. Cada número va acompañado por el nombre la actividad.

En la última columna, aparece una breve descripción de las actividades citadas en la columna anterior.

\section{En la Figura 3:}

En la fila horizontal encontramos letras de la $\mathrm{A}$ a la J, siendo cada una de ellas una sesión de cincuenta minutos.

En la columna de la izquierda aparecen números del 1 al 4, siendo cada uno de ellos el número de actividad dentro de la sesión.

En el caso de la Figura 3, en las casillas resultantes de las intersecciones entre columna y fila encontramos una numeración del 1 al 4 . Esta numeración representa lo siguiente:

1. Actividad comunicativa de la lengua, que se trabaja en esa actividad concreta (recepción, producción, interacción o mediación).

2. Funciones cognitivas del discurso que se ponen en marcha.

3. Dominio de actividad, estudiando el modo en el que se trabajan las formas de hacer, organizar, explicar y argumentar de la propia asignatura (Graz Group, 2016).

4. Géneros, o modos en los que se comunica el conocimiento, entendiendo que bajo los mismos subyacen patrones predecibles y reproducibles (Graz Group, 2016) 


\section{Análisis}

A continuación, se presentan las Figuras 2 y 3 con el análisis de la información recogida en la secuencia didáctica:

\begin{tabular}{|c|c|c|}
\hline \multirow{4}{*}{$\begin{array}{l}\text { SESIÓN A } \\
\text { Characterisation } \\
\text { department needs YOU }\end{array}$} & $\begin{array}{l}\text { 1. TIME MINISTRY: Characterisation } \\
\text { Department }\end{array}$ & \multirow{4}{*}{$\begin{array}{l}\text { El alumnado analizará las caracteristicas básicas de la vida en la Edad Media bajo la } \\
\text { ambientación de llegar a ser agentes del Ministerio del Tiempo. En esta sesión, primero } \\
\text { observarán unas imágenes de la Edad Media en las que tendrán que buscar anacronismos } \\
\text { de forma oral para después describir en detalle, por escrito, otras imágenes con escenas } \\
\text { propias de la Edad Media (oficios, pasatiempos, higiene,etc.). En sus textos se hará énfasis } \\
\text { en el uso del pasado simple y there was/there were. }\end{array}$} \\
\hline & 2. Anachronism & \\
\hline & 3. Pictures from the past & \\
\hline & 4. Snowstorm in the middle ages & \\
\hline \multirow{3}{*}{$\begin{array}{l}\text { SESIÓN B } \\
\text { Tell me how it was }\end{array}$} & 1. Match your memories & \multirow{3}{*}{$\begin{array}{l}\text { Sesión destinada a profundizar en el conocimiento del alumnado sobre las caracteristicas } \\
\text { básicas de la vida en la Edad Media centrando la atención en el aprendizaje de } \\
\text { vocabulario. La sesión comienza con un juego de unir parejas (Ej.: Hombre vendiendo en } \\
\text { un mercado = Comercio). Posteriormente, deberán escoger un tema de los mostrados de } \\
\text { esta época para realizar una infografia sobre el mismo y presentarla oralmente ante la } \\
\text { clase. }\end{array}$} \\
\hline & 2. Infographic & \\
\hline & 3. History Heralds & \\
\hline \multirow{3}{*}{$\begin{array}{l}\text { SESIÓN C } \\
\text { Medieval people }\end{array}$} & 1. Zap it! & \multirow{3}{*}{$\begin{array}{l}\text { La intención es trabajar sobre el vocabulario relacionado con las posiciones sociales } \\
\text { propias de la Edad Media. Por parejas, el alumnado deberá escribir, utilizando el pasado } \\
\text { simple una biografia sobre un tipo de persona de la Edad Media (Ej.: La historia de vida } \\
\text { de un rey). }\end{array}$} \\
\hline & 2. Find your match & \\
\hline & 3. Medieval tales & \\
\hline \multirow{2}{*}{$\begin{array}{l}\text { SESIÓN D } \\
\text { Medieval people II }\end{array}$} & 1. Skip the medieval Word & \multirow{2}{*}{$\begin{array}{l}\text { Comienza la sesión con un juego escrito de repaso de vocabulario utilizando como base el } \\
\text { formato de "pasapalabra". Después, la sesión está orientada a terminar las historias } \\
\text { iniciadas en la sesión anterior sobre los personajes de la Edad Media. }\end{array}$} \\
\hline & 2. Medieval tales II & \\
\hline \multirow{3}{*}{$\begin{array}{l}\text { SESIÓN E } \\
\text { Sharing stories }\end{array}$} & 1. TIC IAC TOE & \multirow{3}{*}{$\begin{array}{l}\text { Esta sesión comenzará con un juego colaborativo de repaso de vocabulario a través } \\
\text { definiciones pero con un formato similar al tres en raya. Iras esto, el alumnado realizará } \\
\text { una corrección entre pares sobre los textos escritos y, una vez realizadas las correcciones, } \\
\text { se leerán en voz alta las historias escritas. }\end{array}$} \\
\hline & 2. The Editor & \\
\hline & 3. Storytelling o'clock & \\
\hline \multirow{3}{*}{$\begin{array}{l}\text { SESIÓN F } \\
\text { Troubadours on duty }\end{array}$} & 1. TIC IAC TOE - Review & \multirow{3}{*}{$\begin{array}{l}\text { Se realizará de nuevo el tres en raya de la sesión anterior, pero esta vez de manera } \\
\text { individual. Posteriormente, se analizarán las caracteristicas de tres de las obras más } \\
\text { relevantes de la literatura anglosajona (Robin Hood, King Arthur y Beowulf) a través de } \\
\text { una actividad que, con un formato puzzle, requerirá que el alumnado por equipos } \\
\text { clasifique y ordene trozos de texto en su historia correspondiente. }\end{array}$} \\
\hline & 2. Once upon a time... & \\
\hline & 3. Let's share & \\
\hline \multirow{3}{*}{$\begin{array}{l}\text { SESIÓN G } \\
\text { Comic me }\end{array}$} & 1. Going to the movies & \multirow{3}{*}{$\begin{array}{l}\text { La sesión comienza con un repaso de las historias vistas el dia anterior a través del } \\
\text { visionado de escenas de su adaptación al cine. En segundo lugar, por parejas, el objetivo } \\
\text { será crear cómics que representen las historias medievales aprendidas. Se valora el uso } \\
\text { del simple past y el vocabulario utilizado hasta el momento. }\end{array}$} \\
\hline & 2. Now the comic & \\
\hline & 3. Check the final version & \\
\hline \multirow{3}{*}{$\begin{array}{l}\text { SESIÓN H } \\
\text { Improve your comic }\end{array}$} & 1. $3^{\prime} 3 \mathrm{P} 3 \mathrm{Q}$ & \multirow{3}{*}{$\begin{array}{l}\text { A través de una actividad de speaking, el alumnado se moverá libremente por la clase } \\
\text { para hacerse preguntas sobre una imagen que se proyecta en la pantalla haciendo uso de } \\
\text { las WH-Questions. Después, se dejará tiempo para terminar los cómics, se realizará } \\
\text { revisión entre pares de los mismos y se expondrán en las paredes de la clase. }\end{array}$} \\
\hline & 2. Quick review & \\
\hline & 3. Supervisors' certificate & \\
\hline SESIÓN I & 1. Scoot day & $\begin{array}{l}\text { Se utilizará la sesión para realizar una actividad conocida como Scoot. En esta actividad, el } \\
\text { alumnado deberá ir moviéndose por la clase buscando y respondiendo a una serie de } \\
\text { preguntas. Para ello tendrá que recordar todo lo aprendido previamente y escribirlo en su } \\
\text { ficha de respuestas. }\end{array}$ \\
\hline \multirow{3}{*}{ SESIÓN J } & 1. Back to the past & \multirow{3}{*}{$\begin{array}{l}\text { Se inicia la sesión con un Daily Routine ambientado en la Edad Media, asi, a preguntas } \\
\text { como What did you do this weekend? deberán contestar con una historia ambientada en } \\
\text { la época. Por último, como prueba final para acceder al Ministerio del Tiempo el } \\
\text { alumnado deberán trabajar en parejas y escribir las preguntas y respuestas que desde el } \\
\text { Ministerio del tiempo se le haria a un candidato a Agente. De este modo, el candidato } \\
\text { deberia hacerse pasar por un personaje de la Edad Media y ser capaz de responder a } \\
\text { preguntas sobre su vida sin salirse de las caracteristicas propias de esa época y persona. } \\
\text { Terminadas las entrevistas, se hará un roleplay con las mismas para que la clase decida si } \\
\text { los condidatos pasan la prueba. }\end{array}$} \\
\hline & 2. Now hiring! & \\
\hline & 3. What's on my head? & \\
\hline
\end{tabular}

Figura 2. Relación entre sesiones y actividades dentro de la secuencia didáctica. 


\begin{tabular}{|c|c|c|c|c|c|c|c|c|c|c|}
\hline$S$ & $A$ & $B$ & $C$ & $D$ & $E$ & $F$ & $G$ & $\boldsymbol{H}$ & $I$ & $\boldsymbol{J}$ \\
\hline 1 & $\begin{array}{l}1 . \\
\text { Comprensión } \\
\text { oral }\end{array}$ & $\begin{array}{l}\text { 1. Interacción } \\
\text { oral } \\
\text { 2.Classify } \\
\text { 3. Organizar } \\
\text { 4.Clasificación }\end{array}$ & $\begin{array}{l}\text { 1.Comprensión } \\
\text { oral } \\
\text { 2.Classify } \\
\text { 3. Organizar } \\
\text { 4.Simulación }\end{array}$ & $\begin{array}{l}\text { 1.Comprensión } \\
\text { lectora } \\
\text { 2.Define } \\
\text { 3.Explicar } \\
\text { 4.Definición }\end{array}$ & $\begin{array}{l}\text { 1.Comprensión } \\
\text { lectora } \\
\text { 2.Define } \\
\text { 3.Explicar } \\
\text { 4.Definición }\end{array}$ & $\begin{array}{l}\text { 1.Comprensión } \\
\text { lectora } \\
\text { 2.Define } \\
\text { 3.Explicar } \\
\text { 4.Definición }\end{array}$ & $\begin{array}{l}\text { 1. Recepción } \\
\text { audiovisual } \\
\text { 2.Define } \\
\text { 3.Explicar } \\
\text { 4.Explicación }\end{array}$ & $\begin{array}{l}\text { 1. Interacción oral } \\
\text { 2.Describe } \\
\text { 3. Organizar } \\
\text { 4.Conversación } \\
\text { informal }\end{array}$ & $\begin{array}{l}\text { 1.Comprensión } \\
\text { lectora } \\
\text { 2.Define } \\
\text { 3.Explicar } \\
\text { 4.Prueba de } \\
\text { evaluación }\end{array}$ & $\begin{array}{l}\text { 1.Interacción } \\
\text { oral } \\
\text { 2.Describe } \\
\text { 3. Organizar } \\
\text { 4.Daily Routine }\end{array}$ \\
\hline 2 & $\begin{array}{l}\text { 1. Interacción } \\
\text { oral } \\
\text { 2.Classify } \\
\text { 3.Organizar } \\
\text { 4.Comparación }\end{array}$ & $\begin{array}{l}\text { 1.Interacción } \\
\text { oral. } \\
\text { Producción } \\
\text { escrita } \\
\text { 2.Report } \\
\text { 3.Hacer } \\
\text { 4.Infografia }\end{array}$ & $\begin{array}{l}\text { 1.Interacción } \\
\text { oral } \\
\text { 2.Classify } \\
\text { 3.Organizar } \\
\text { 4.Unión de } \\
\text { parejas }\end{array}$ & $\begin{array}{l}\text { 1.Producción } \\
\text { escrita. } \\
\text { Interacción oral } \\
\text { 2.Explore } \\
\text { 3.Razonar } \\
\text { 4.Biografia }\end{array}$ & $\begin{array}{l}\text { 1.Mediación de } \\
\text { texto } \\
\text { 2.Evaluate } \\
\text { 3.Razonar } \\
\text { 4.Evaluación } \\
\text { entre pares }\end{array}$ & $\begin{array}{l}\text { 1.Comprensión } \\
\text { lectora. } \\
\text { Interacción oral } \\
\text { 2.Classify } \\
\text { 3. Organizar } \\
\text { 4.Puzzle de } \\
\text { textos }\end{array}$ & $\begin{array}{l}\text { 1.Produccín } \\
\text { escrita. } \\
\text { Interacción oral } \\
\text { 2.Report } \\
\text { 3. Hacer } \\
\text { 4.Cómic }\end{array}$ & $\begin{array}{l}\text { 1.Producción } \\
\text { escrita. } \\
1 \text { Interacción oral } \\
\text { 2.Evaluate } \\
\text { 3.Razonar } \\
\text { 4.Autoevaluación }\end{array}$ & & $\begin{array}{l}\text { 1.Producción } \\
\text { escrita. } \\
\text { Interacción oral } \\
\text { 2.Explore } \\
\text { 3.Razonar } \\
\text { 4.Entrevista }\end{array}$ \\
\hline 3 & $\begin{array}{l}\text { 1. Comprensión } \\
\text { lectora e } \\
\text { interacción oral } \\
\text { 2.Describe } \\
\text { 3. } \begin{aligned} \text { ganizarar } \\
\text { 4.Descripción }\end{aligned}\end{array}$ & $\begin{array}{l}\text { 1.Producción } \\
\text { oral } \\
\text { 2.Report } \\
\text { 3.Hacer } \\
\text { 4.Infografia }\end{array}$ & $\begin{array}{l}\text { 1.Producción } \\
\text { escrita. } \\
\text { Interacción } \\
\text { oral. } \\
\text { 2.Explore } \\
\text { 3.Razonar } \\
\text { 4.Biografia }\end{array}$ & & $\begin{array}{l}\text { 1. Expresión } \\
\text { oral } \\
\text { 2. Report } \\
\text { 3. Hacer } \\
\text { 4. Narración }\end{array}$ & $\begin{array}{l}\text { 1.nteracción } \\
\text { oral } \\
\text { 2.Define } \\
\text { 3.Explicar } \\
\text { 4.Tabla de } \\
\text { resultados }\end{array}$ & $\begin{array}{l}\text { 1. Mediación de } \\
\text { texto } \\
\text { 2.Evaluate } \\
\text { 3.Razonar } \\
\text { 4.RŔbrica de } \\
\text { autoevaluación }\end{array}$ & $\begin{array}{l}\text { 1.Mediación de } \\
\text { texto escrito } \\
\text { 2.Evaluate } \\
\text { 3.Razonar } \\
\text { 4.Rúbrica de } \\
\text { evaluación entre } \\
\text { pares }\end{array}$ & & $\begin{array}{l}\text { 1.Interacción } \\
\text { oral. } \\
\text { 2.Describe } \\
\text { 3.Organizar } \\
\text { 4.Adivinanza }\end{array}$ \\
\hline 4 & $\begin{array}{l}\text { 1.Producción } \\
\text { escrita. } \\
\text { Interacción oral } \\
\text { 2.Evaluate } \\
\text { 3. Razonar } \\
\text { 4.Reflexión } \\
\text { personal }\end{array}$ & & & & & & & & & \\
\hline
\end{tabular}

Figura 3. Relación entre sesiones y actividades dentro de la secuencia didáctica en relación a: 1. Actividad comunicativa de la lengua; 2. Funciones cognitivas del discurso; 3. Dominio de actividad; 4. Género.

\section{Interpretación}

La información presente en la Figura 3 se interpreta en los siguientes apartados. Para favorecer la comprensión, los apartados se muestran organizados en las categorías analizadas en dicha figura.

\subsection{Actividades comunicativas de la lengua}

En la secuencia didáctica se ha sustituido el modelo de las cuatro destrezas por el indicado por el CEFR (2018) de actividades de la lengua. Una de las primeras cuestiones a destacar de la Figura 3 es que en el número 1 de las casillas, en ocasiones, aparece más de una actividad de la lengua. Esto se debe a que la naturaleza de algunas actividades permite que se dé más de una al mismo tiempo. Según la Figura 3, esta situación ocurre en los ejercicios en equipo o parejas, donde además de poner en marcha la actividad de la lengua que requiera la actividad (ej.: producción escrita) siempre aparece la interacción oral necesaria para que los miembros del equipo se pongan de acuerdo hablando entre ellos. El CEFR (2018) nombra esta acción como "goal-oriented co-operation". Esto ocurre en 8 de las 28 actividades totales, lo que supone una presencia de alrededor de un $29 \%$.

Con la intención de aportar una visión más detallada, la Tabla 1 muestra el número de veces que se han repetido las diferentes actividades y, a continuación, se muestra el Gráfico 1 con la representación visual de dichos datos. 


\begin{tabular}{|c|c|c|c|c|c|c|c|}
\hline \multicolumn{3}{|c|}{ RECEPCIÓN } & \multicolumn{2}{|c|}{ PRODUCCIÓN } & \multicolumn{2}{|c|}{ INTERACCIÓN } & MEDIACIÓN \\
\hline comprension & Conprension & Audiovisual & oral & Escrita & oral & Escrita & De texto \\
\hline 2 & 6 & 1 & 1 & 7 & 16 & 0 & 3 \\
\hline
\end{tabular}

Tabla 1. Resultados de uso de las actividades de la lengua.

\section{ACTIVIDADES DE LA LENGUA}

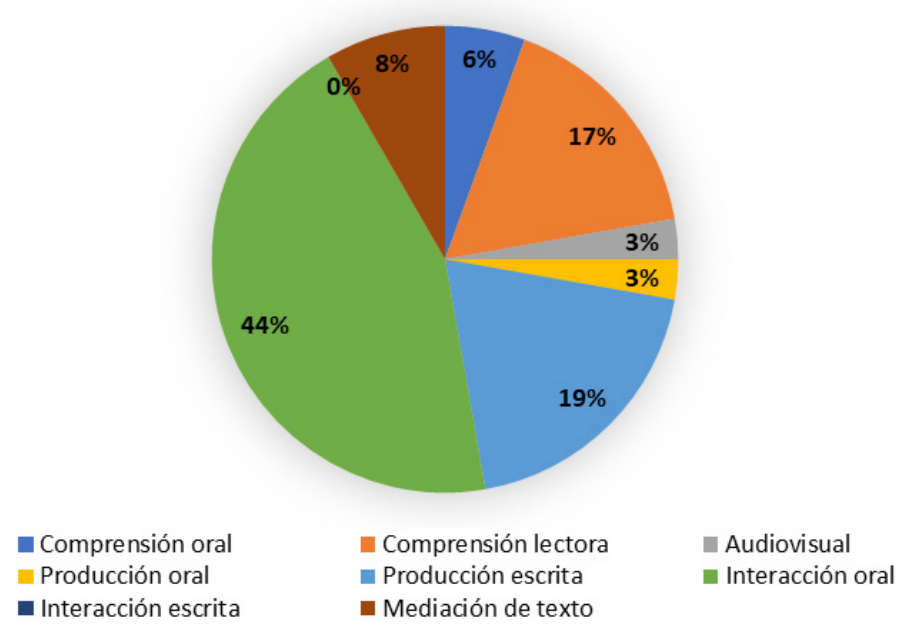

Gráfico 1. Resultados de uso de actividades de la lengua.

Como observamos tanto en la Tabla 1 como en el Gráfico 1, la interacción oral es la actividad de la lengua predominante, obteniendo un resultado mucho mayor que el resto (40\%). A lo largo de la secuencia didáctica se presentan muchas actividades en las que el alumnado debe poner en marcha diferentes tipos de interacción oral. Se observa que el tiempo de habla exclusiva por parte del docente es inexistente, la relación entre docente-alumnado que fomenta las propuestas se relaciona con un formato de preguntas y respuestas, conversaciones o intercambio de información. Entre el propio alumnado, como se indicaba anteriormente, se busca el uso del idioma para cooperar en la realización conjunta de actividades o la construcción colaborativa de significados. De manera contrapuesta, observamos que se reduce el tiempo de exposición del alumnado a la simple escucha o comprensión oral (3\%) propia de modelos más clásicos como las lecciones magistrales. Los medios audiovisuales están presentes (3\%) pero de un modo anecdótico, ya que el acento está en la interacción. La interacción escrita no aparece representada en esta propuesta ( $0 \%$ ) ya que el formato de la secuencia didáctica no ha recurrido a este tipo de actividad.

Dado que nos encontramos en $5^{\circ}$ de Primaria y este alumnado lleva, como mínimo, cuatro años de trabajo previo con el idioma, el desarrollo de actividades relacionadas con la comprensión lectora $(17 \%)$ y producción escrita $(18 \%)$ también ocupan un papel relevante. 
Por último, existen varias actividades basadas en la corrección entre pares o la autocorrección. Este tipo de actividades favorecen que el que el alumnado reflexione consigo mismo o explique a sus compañeros/as los fallos lingüísticos que tiene, cómo mejorarlo y aclarar entre sí conceptos que hayan podido generar malentendidos. Atendiendo a esta situación, dichas actividades han sido etiquetadas como mediación de texto, suponiendo un $8 \%$ del total.

\subsection{Funciones cognitivas del discurso y dominio de actividad}

En este apartado analizamos la Figura 4 atendiendo a la presencia de las diferentes CDF (DaltonPuffer, 2013). Como recordamos, esta autora presentaba 7 tipos distintos de CDF, aunque ella misma señalaba que estas etiquetas resultan semánticamente opacas. Asimismo, estas etiquetas no han sido concebidas para ser aplicadas en la propia asignatura de Inglés como lengua extranjera, sino para contextos de integración de contenido y lengua. Debido a esta realidad citada anteriormente con respecto a que los conceptos marcados por las CDF son algo difusos, no son cerrados y se ajustan a las realidades sobre los que se aplican, lo que aquí se presenta es una interpretación personal de las etiquetas asociadas a las CDF presentes en las actividades en la secuencia didáctica.

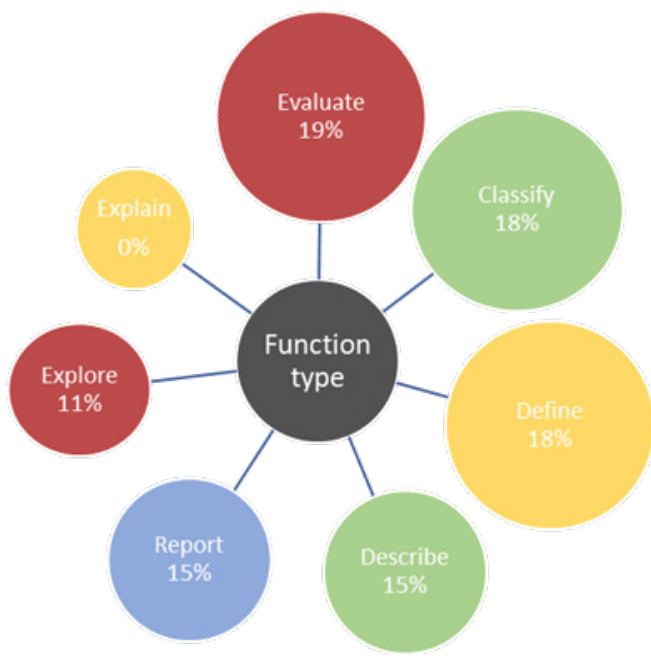

Figura 4. Resultados funciones cognitivas del discurso y dominios de actividad.

En el caso de la Figura 4 los tipos de CDF no aparecen presentados en orden. Para leer esta figura es necesario comenzar en la parte superior y seguir la dirección de las agujas del reloj. Observamos entonces los 7 tipos de CDF, pero ordenados de mayor a menor presencia en la secuencia didáctica.

Lo primero que resaltamos es la existencia de cierto equilibrio entre ellas, es decir, todas, a excepción de una, están presentes de un modo similar. Analizando dicho equilibrio un poco más en detalle observamos que las destrezas cognitivas más repetidas son evaluate, classify $y$ define. Podemos considerar entonces que, en esta ocasión, se han utilizado fundamentalmente estas herramientas para hacer accesible y visibilizar el conocimiento adquirido por el alumnado. El peso del lenguaje recae sobre la reflexión en el propio aprendizaje a través de actividades de autoevaluación o revisión entre pares. Por otro lado, classify aparece repetida en el mismo número de ocasiones que 
define. Si atendemos a la Figura 4 comprobamos que este tipo de CDF se utilizan fundamentalmente en las primeras actividades de cada sesión. Coincidiendo así con las actividades de warm up o de activación de conocimientos previos.

En un segundo grupo aparecen describe and report. Ambas están presentes en las actividades centrales de la sesión, asociándose a actividades más complejas que exigen un uso más cuidado y reflexivo del lenguaje.

En el siguiente nivel encontraríamos explore. En ocasiones ha resultado complicado diferenciar entre explore y report, surgiendo dudas sobre cuál asociar a ciertos tipos de actividades. Finalmente, se ha optado por relacionar la actividad de report con aquellas actividades donde el alumnado debía trabajar sobre textos que ya se le ofrecían de antemano (ej.: Crear un cómic a partir de una historia conocida como la de Robin Hood; presentar la información observada en una fotografía en forma de infografía). Por otro lado, se ha asociado la palabra explore con el desarrollo de textos creativos en los que el alumnado no contaba con referencias y debía por tanto especular, simular o tomar otras perspectivas. Estos verbos, en el modelo de Dalton-Puffer (2013), aparecen asociados a la idea de explore y, aunque esta autora los asocia a contenidos científicos, en esta investigación se han ajustado al contexto en el que nos encontramos.

El único elemento sin representación sería explain. En la asignatura de Inglés, planteada desde nuestra perspectiva, se da prioridad a otras cuestiones que buscan utilizar el idioma como núcleo del trabajo, más que como herramienta para realizar explicaciones complejas. Posiblemente, este tipo de CDF estaría más cerca de asignaturas científicas. Si bien es cierto que, de resultar relevante, podría incluirse en una secuencia didáctica si partiéramos de preguntas que favorecieran la investigación con respecto al propio idioma (ej.: Explica por qué se utiliza simple past en esta historia), en esta ocasión esta opción no ha sido considerada.

Otro detalle a analizar de la Figura 4 son los colores de la misma. Estos tienen relación con los dominios de actividad por los cuales las CDF se asocian a los modos de hacer (círculos azules), organizar (verdes), explicar (amarillos) y razonar (rojos) de cada asignatura. Igualmente, a través del Gráfico 2 observamos balance entre los mismos:

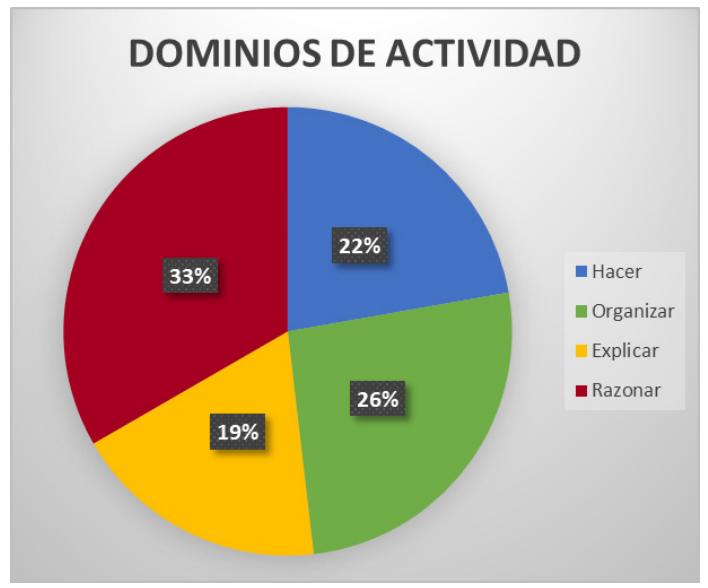

Gráfico 2. Resultados de uso de los dominios de actividad. 
Por otra parte, al analizar su presencia en la Figura 3 y asociarla a las CDF podemos decir que la asignatura de Inglés en nuestro caso "razona" fundamentalmente a través de la evaluación; la información se "organiza" en base a clasificaciones trabajando con categorías, unión, contraste entre diferentes elementos...; se "explica" partiendo del uso de definiciones; finalmente, se "hace" a través de informes o reportes.

\subsection{Género}

Por último, en la Figura 5 se presenta una nube de conceptos en la que se han puesto en común aquellas palabras utilizadas para describir el género empleado en cada actividad. Aquellas palabras que más se repiten aparecen más grandes, siendo las que menos se repiten las que aparecen más pequeñas. Esto nos permite hacernos una idea de aquellos géneros que, en el caso de esta asignatura entendida desde este proceso, tienen mayor presencia.

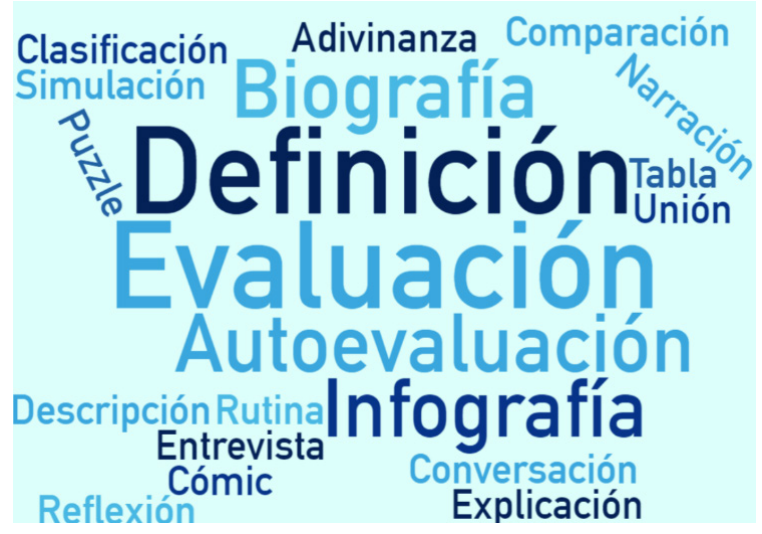

Figura 5. Nube de palabras con la frecuencia de los géneros utilizados.

Es importante recordar que los géneros, como construcciones sociales que son, también son abiertos y pueden tener distintos tamaños y pueden englobar a otros más pequeños dentro de sí mismos. Los que aquí se plantean son los considerados por la investigación desde una perspectiva personal. De este modo, deben entenderse como un ejemplo o una selección de algunos que podrían utilizarse en un aula de lengua extranjera.

\section{Conclusiones}

Los datos presentes en este artículo muestran la posibilidad de aplicar con éxito el modelo PTL en contextos específicos de idioma sin tener que recurrir a la integración de otro contenido para aportar más significados. La propia asignatura parece contar con el potencial de encontrar contextos significativos que amplíen la experiencia del alumnado. Se abre así la puerta a la posibilidad de lograr mejoras en el área asegurándonos que, en su propio terreno, el espacio desde el que se trabaja está cuidado y, por tanto, no resulte imprescindible expandir su campo de aplicación a otras áreas. 
Como indica Do Coyle (2015) esta mirada reta el modelo predominante de aprendizaje de idiomas centrado en el valor de un desarrollo cronológico de la gramática. Desde esta experiencia se valora la creación de un camino alternativo que identifique la lengua que los estudiantes necesitarán, no en un contexto de integración, sino en el aprendizaje del inglés. La gramática deja entonces de ser el filtro de organización y planteamiento de objetivos. En este análisis hemos visto cómo la interacción oral se adelanta y encuentra un hueco predominante en el aula. Tal y como establece el CEFR (2018), la interacción oral resulta fundamental para el aprendizaje y es importante darle el protagonismo que necesita. Sobre todo, en el caso de la interacción oral, considerada como el origen de la lengua dadas sus funciones interpersonales, colaborativas y transaccionales. Así, podemos determinar que, al aplicar un modelo como el PTL en la práctica educativa, el uso oral del idioma desde la interacción se verá potenciado. Igualmente, la presencia de esta actividad de la lengua, ya que nos encontramos en $5^{\circ}$ de primaria, se completa con otras. El alumnado comienza a estar preparado para asentar los conocimientos orales y aplicarlos a otros más conscientes relacionados con la lectura y la escritura y, de ahí, que este tipo de cuestiones sigan en relevancia a la interacción oral en la secuencia.

Por otro lado, el estudio de las actividades de la lengua y las CDF presentes en este análisis determina que este modelo incrementa el protagonismo de los estudiantes en su proceso de aprendizaje, dotándoles de espacios para usar la lengua como herramienta de reflexión consciente. Pudiendo aprender de sus errores y disfrutar del idioma dentro de un clima de aula positivo, adaptable a las particularidades y no encorsetado en la búsqueda de la eficiencia gramatical. Se genera un lugar donde poder verbalizar el conocimiento y, en consecuencia, lograr ese aprendizaje profundo o deep learning que sirva de nexo entre los dos elementos fundamentales del proceso, conceptual continuum y communicating continuum.

En cualquier caso, esta experiencia es una aproximación hacia los posibles cambios que este tipo de metodologías puede aportar a la materia que nos ocupa. Aun así, resulta difícil comparar estos resultados con los obtenidos en otros estudios, dado que, a día de hoy, no se conocen muchos entornos donde estas reflexiones se hayan llevado a análisis. Por tanto, el objetivo último de este artículo es animar a que la investigación en esta área siga creciendo y se le dedique el espacio y el tiempo necesario para poder avanzar en la buena dirección. 


\section{Referencias}

Bernaus, M., Furlong, Á., Jonckheere, S. y Kervran, M. (2011). Plurilingualism and pluriculturalism in content-based teaching: A training kit. Council of Europe Publishing.

Consejo de Europa. (2002). Marco común europeo de referencia para las lenguas: Aprendizaje, enseñanza, evaluación. Anaya.

Council of Europe (2018). Common European Framework of Reference for Languages: Learning, teaching, assessment companion volume with new descriptors. Consejo de Europa.

Coyle, D., Hood, P. y Marsh, D. (2010). CLIL: Content and language integrated learning. Cambridge University Press.

Coyle, D. (2015). Strengthening integrated learning: Towards a new era for pluriliteracies and intercultural learning terms of creating a dynamic theoretical and practice-oriented foundation for the development of Content and Language Integrated Learning (CLIL) across. LACLIL, 8(2), 84-103. https://doi.org/10.5294/laclil.2015.8.2.2

Dalton-Puffer, C. (2013). A construct of cognitive discourse functions for conceptualising content-language integration in CLIL and multilingual education. European Journal of Applied Linguistics, 1(2), 216-253. https://10.1515/eujal-2013-0011

Graz Group. (2016). The pluriliteracies wheel. Creating deep learning tasks by enabling students to language subject specific activities. https://pluriliteracies.ecml.at/Portals/54/img/pluriliteracies-wheel.jpg

Halbach, A. (2019). Inglés en tiempos de CLIL: propuesta para una nueva metodología para las clases de inglés. Padres y Maestros, 378, 6-10.

Meyer, O. y Coyle, D. (2017). Pluriliteracies teaching for learning: conceptualizing progression for deeper learning in literacies development. European Journal of Applied Linguistics, 5(2), 199-222. https://doi.org/10.1515/eujal-2017-0006

Meyer, O., Coyle, D., Halbach, A. y Schuck, K. (2015). A pluriliteracies approach to content and language integrated learning - mapping learner progressions in knowledge construction and meaning- making. Language, Culture and Curriculum, 28(1), 41-57. https://doi.org/10.1080/07908318.2014.1000924.

Meyer, O., Coyle, D., Imhof, M. y Connolly, T. (2018). Beyond CLIL : Fostering student and teacher engagement for personal growth and deeper learning. En J. de D. Martinez Agudo (Ed.), Emotions in second language teaching: Advances in theory and research, and teacher education (pp. 277-297). Springer.

Meyer, O., Halbach, A. y Coyle, D. (2015). A pluriliteracies approach to teaching for learning: Putting a pluriliteracies approach into practice. https://pluriliteracies.ecml.at/

Meyer, A., Rose, D. H. y Gordon, D. (2014). Universal design for learning: Theory and practice. https://doi.org/10.1016/j.learninstruc.2016.01.008

Pérez Cañado, M. L. (2016). From the CLIL craze to the CLIL conundrum: Addressing the current CLIL controversy. Bellaterra Journal of Teaching and Learning Language and Literature, 9(1), 9-31. https://doi.org/10.5565/rev/jtl3.667

Schleppegrell, M. J. (2001). Linguistic features of the language of schooling. Linguistics and Education, 12(4), 431-459. https://doi.org/10.1016/S0898-5898(01)00073-0

Wang, M.-T., Fredricks, J. A., Ye, F., Hofkens, T. L. y Linn, J.S. (2016). The math and science engagement scales: Scale development, validation, and psychometric properties. Learning and Instruction, 43, 16-26. https://doi.org/10.1016/j.learninstruc.2016.01.008 